\title{
A novel SOX10 nonsense mutation in a patient with Kallmann syndrome and Waardenburg syndrome
}

Tetsuji Wakabayashi, Akihito Takei, Nobukazu Okada, Miki Shinohara, Manabu Takahashi, Shuichi Nagashima, Kenta Okada, Ken Ebihara and Shun Ishibashi

Division of Endocrinology and Metabolism, Department of Internal Medicine, School of Medicine, Jichi Medical University, Shimotsuke, Tochigi, Japan

Correspondence should be addressed to S Ishibashi

Email

ishibash@jichi.ac.jp

\section{Summary}

The underlying genetic drivers of Kallmann syndrome, a rare genetic disorder characterized by anosmia and hypogonadotropic hypogonadism due to impairment in the development of olfactory axons and in the migration of gonadotropin-releasing hormone (GNRH)-producing neurons during embryonic development, remain largely unknown. SOX10, a key transcription factor involved in the development of neural crest cells and established as one of the causative genes of Waardenburg syndrome, has been shown to be a causative gene of Kallmann syndrome. A 17-year-old male patient, who was diagnosed with Waardenburg syndrome on the basis of a hearing impairment and hypopigmented iris at childhood, was referred to our department because of anosmia and delayed puberty. As clinical examination revealed an aplastic olfactory bulb and hypogonadotropic hypogonadism, we diagnosed him as having Kallmann syndrome. Incidentally, we elucidated that he also presented with subclinical hypothyroidism without evidence of autoimmune thyroiditis. Direct sequence analysis detected a nonsense SOX10 mutation (c.373C >T, p.Glu125X) in this patient. Since this nonsense mutation has never been published as a germline variant, the SOX10 substitution is a novel mutation that results in Kallmann syndrome and Waardenburg syndrome. This case substantiates the significance of SOX10 as a genetic cause of Kallmann syndrome and Waardenburg syndrome, which possibly share a common pathway in the development of neural crest cells.

\section{Learning points:}

- Kallmann syndrome and Waardenburg syndrome possibly share a common pathway during neural crest cell development.

- SOX10, a key transcription factor involved in the development of neural crest cells, is a common causative gene of Kallmann syndrome and Waardenburg syndrome.

- Careful evaluation about various phenotypic features may reveal the unknown genetic drivers of Kallmann syndrome.

\section{Background}

Kallmann syndrome (KS) is a rare hereditary disorder characterized by congenital hypogonadotropic hypogonadism with hyposmia/anosmia due to impairment in the development of olfactory axons and in the migration of gonadotropin-releasing hormone (GNRH)-producing neurons during embryonic development. While more than 30 genes have been implicated in KS to date, including ANOS1 (KAL1), 
NSMF, FGF8, FGFR1, FGF17, IL17RD, DUSP6, SPRY4, GLCE, FLRT3, PROK2, PROKR2, HS6ST1, CHD7, WDR11, SEMA3A, PLXNA1, SEMA7A, SEMA3E, DCC, NTN1, AMH, AMHR2, NDNF, TUBB3, KLB, HESX1, SOX10, and $F E Z F 1$, mutations in these genes never account for all the genetic etiology (1). KS can be associated with several additional non-olfactory or non-reproductive symptoms, including hearing loss, which occurs in approximately $5 \%$ of KS individuals. While some cases of KS with hearing loss are linked to mutations in ANOS1, FGFR1, FGF8, IL17RD, and CHD7, the majority have unknown genetic features (2).

In 2013, Pingault et al. reported a high frequency of SOX10 mutations in KS patients with hearing loss (3). SOX10 was initially identified as a gene mutated in patients with Waardenburg syndrome (WS). This syndrome is also an uncommon genetic disorder that manifests with congenital sensorineural deafness and facial dysmorphic features, represented by dystopia canthorum (wide distance between the inner corners of the eyes), and abnormal pigmentation of the hair, skin, and iris. Depending on phenotypic variations, WS is classified into four subtypes, namely WS1-WS4. In brief, while WS2 is not associated with any additional features, WS1, WS3, and WS4 are associated with dystopia canthorum, limb abnormality, and Hirschsprung disease (HD), respectively. WS is considered to be caused by abnormal neural crest differentiation during embryonic development, and several mutations of six genes involved in the development of neural crest cells, including $P A X 3$, $M I T F, E D N 3, E D N R B, S N A I 2$, and SOX10, have been reported (4)

SOX10, which has been shown to be involved in WS2 and WS4 (5), is a transcription factor essential for neural crest and peripheral nervous system development and differentiation. Since SOX10 mutations have been primarily focused as a causative gene of WS, its relevance to the etiology of KS has not been established. Pingault et al. incidentally found a high frequency of olfactory bulb agenesis in WS patients with SOX10 mutations, and they revealed that SOX10-null mice showed an absence of olfactory ensheathing cells along the olfactory nerve pathway accompanied by a deficiency in GnRH cell migration (3). Therefore, SOX10 has been recognized as a common gene involved in both $\mathrm{KS}$ and WS.

Here, we report a patient with KS, congenital deafness, and a unilateral hypopigmented iris, who carries a novel SOX10 nonsense mutation.

\section{Case presentation}

A 17-year-old male patient was referred for delayed puberty and anosmia. He was born to nonconsanguineous Japanese parents after a normal pregnancy. He presented with a right hypopigmented iris without poor eyesight at birth. He was diagnosed with deafness at 2.5 years; while he was suspected of having WS, he received only hearing aids and speech therapy. His mental development was normal. At 16 years of age, he noticed anosmia by himself, and an intravenous olfaction test using prosultiamine (Alinamin test), conducted at another hospital, revealed no response. Together with coexisting delayed puberty, he was suspected of having KS and visited our hospital.

\section{Investigation}

His height was $171.9 \mathrm{~cm}$ and his body weight was 54.3 kg. Physical examination revealed a right unilateral blue iris (Fig. 1A), thin body hair, no voice breaking, a micropenis, and impalpable testes. The ophthalmoscopy test revealed that his right fundus was red colored (Fig. $1 B)$; however, his visual acuity and visual field were normal. The audiogram test revealed that his right and left average hearing levels were above $90 \mathrm{~dB}$ and 100 $\mathrm{dB}$, respectively, which suggested that he had severe bilateral hearing loss. Dystopia canthorum, pigmentation defects in the skin containing hair, limb anomaly, and gastrointestinal problems suggestive of $\mathrm{HD}$, which are

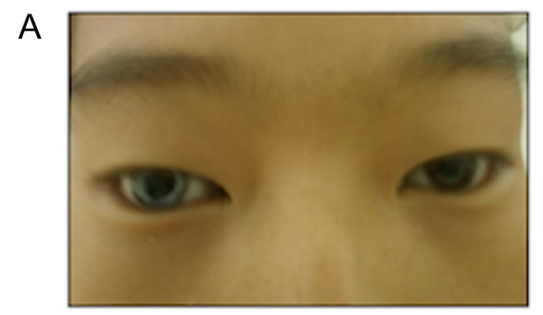

B

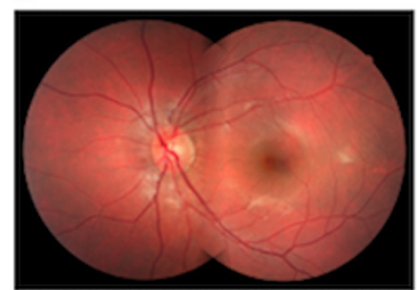

left

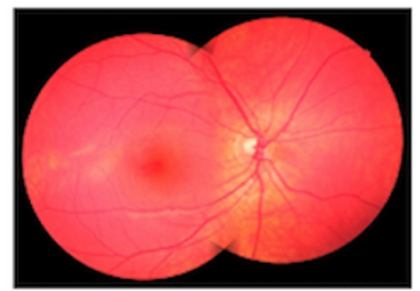

right
Figure 1

(A) Hypopigmentation of the right iris. (B). Representative images of the ophthalmoscopy test. The right panel shows the red colored fundus of this patient. 
representative symptoms of WS, were absent. Further, cleft lip/palate, shortened digits, missing teeth, absence of one kidney, and mirror movements, which are representative complications of KS, were also absent.

Endocrine studies revealed the testosterone level ( 0.18 $\mathrm{ng} / \mathrm{mL}$; reference range: 1.91-8.71), and gonadotropin levels including FSH $(<1.0 \mathrm{mU} / \mathrm{ml}$; reference range (RR): 2.9-10.8) and LH $(<0.2 \mathrm{mU} / \mathrm{ml}$; RR: 0.8-4.2) were very low, with weak and delayed response to a single GNRH stimulation (Table 1). Continuous stimulation with GNRH (administered for six consecutive days) induced a normal response to gonadotropin, indicating the persistent function of the pituitary. The rise in the plasma testosterone level after 3 days of HCG stimulation was insufficient, suggesting the complication of secondary testicular dysfunction. A high level of TSH $(7.56 \mu \mathrm{U} /$ $\mathrm{ml}$; RR: 0.40-3.60) with normal levels of thyroid hormones, including FT3 (2.95 pg/ml; RR: 2.25-4.53) and FT4 (1.08 ng/dl; RR: 0.97-1.89), indicated subclinical hypothyroidism, however, there was an absence of clinical signs characteristic of autoimmune thyroiditis, including thyroid enlargement and anti-thyroid peroxidase and antithyroglobulin antibodies. Ultrasonography showed that the thyroid presented neither swelling nor atrophy but contained some cysts in the lower part. Other hormone levels, such as ACTH, cortisol, GH, and PRL, were grossly normal. His bone age was delayed ( 14 years of age) and his epiphyseal plates had never closed. CT scan showed two small testes in the scrotum, suggesting an absence of cryptorchidism. MRI showed an aplastic olfactory bulb, whereas no abnormalities were detected around the hypothalamus and pituitary (Fig. 2).

Therefore, we diagnosed him as having both KS and WS, and suspected that a SOX10 mutation was responsible for the clinical manifestations. Indeed, direct sequence analysis identified a nonsense mutation (c.373C $>\mathrm{T}$,

Table 1 Baseline patient endocrine data.

\begin{tabular}{l} 
Hormone \\
\hline Testosterone $(\mathrm{ng} / \mathrm{ml})$ \\
LH $(\mathrm{mU} / \mathrm{ml})$ \\
FSH $(\mathrm{mU} / \mathrm{ml})$ \\
$\mathrm{GH}(\mathrm{ng} / \mathrm{ml})$ \\
IGF-1 $(\mathrm{ng} / \mathrm{ml})$ \\
ACTH $(\mathrm{pg} / \mathrm{ml})$ \\
Cortisol $(\mu \mathrm{g} / \mathrm{dl})$ \\
Prolactin $(\mathrm{ng} / \mathrm{ml})$ \\
TSH $(\mu \mathrm{ml} / \mathrm{ml}$ \\
Free T3 $(\mathrm{pg} / \mathrm{ml})$ \\
Free T4 $(\mathrm{ng} / \mathrm{dl})$
\end{tabular}

\begin{tabular}{c}
\hline Patient \\
\hline 0.18 \\
$<0.2$ \\
$<1.0$ \\
1.0 \\
295 \\
24.2 \\
17.1 \\
13.1 \\
7.56 \\
2.95 \\
1.08 \\
\hline
\end{tabular}

\begin{tabular}{c}
\hline Reference values $^{*}$ \\
\hline $1.91-8.71$ \\
$0.8-4.2$ \\
$2.9-10.8$ \\
$0.07-18.70$ \\
$142-540$ \\
$12.3-33.3$ \\
$4.0-13.0$ \\
$4.3-13.7$ \\
$0.40-3.60$ \\
$2.25-4.53$ \\
$0.97-1.89$
\end{tabular}

*Reference values in age-matched Japanese men.
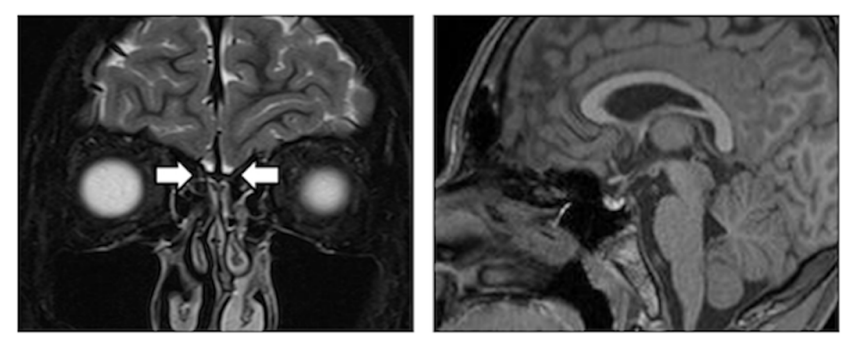

Figure 2

Representative images of brain MRI . The arrows indicate the aplastic olfactory bulb (left panel). No obvious abnormalities were noted in the hypothalamus or pituitary (right panel).

p.Glu125X) in SOX10 (Fig. 3). The first exon in the SOX10 coding region had a heterozygous mutation of replacement of the 373rd nucleotide, $\mathrm{C}$ by T, resulting in replacement of the 123rd amino acid glutamic acid codon with a stop codon, which possibly terminated the translation. This variant was classified as pathogenic based on the ACMG/ AMP guidelines (6) The patient's parents, older sister, and younger brother did not consent to genetic analysis of SOX10, however, they appeared phenotypically normal, and there was no familial occurrence of pubertal delay, cryptorchidism, deafness, and olfactory defects.

\section{Genetic sequencing methodology}

After obtaining written informed consent, genomic DNA was extracted from the peripheral leukocytes of the patient using the QiaAmp Blood Kit (Qiagen). The following primers and annealing temperatures were used to amplify SOX10 gene fragments covering the coding exons (exon 3-5) through PCR as previously described (7): exon 3, 5'-GTTGGACTCTTTGCGAGGAC-3' and 5'-ATCCACCCGAAGCTAGAGG-3' $\left(58^{\circ} \mathrm{C}\right)$; exon 4, 5'-AGCCCCTCTGCTGTCCTCT-3' and 5'-CACCCTCAG CTCTGTCATCA-3' $\left(60^{\circ} \mathrm{C}\right)$; and exon 5, 5'-CTAACC TGCTTCCCCCTTG-3' and 5'-CAAGGAACAGGG CACACAG-3' $\left(58^{\circ} \mathrm{C}\right)$. Sequences of PCR products were obtained using an ABI PRISM 310 Genetic Analyzer

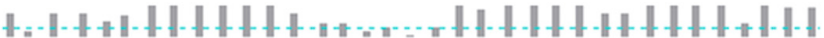
$A G G A A G C T C G C G G A C T A G T A C C C G C A C C T G C A C A A$

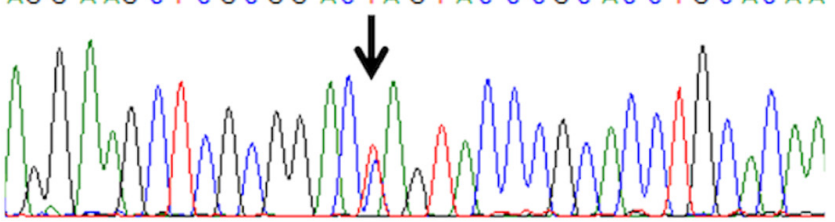

Figure 3

The chromatograms show the partial sequence of exon 3 in this patient. The arrow indicates the c.373C $>$ T heterozygous mutation. 

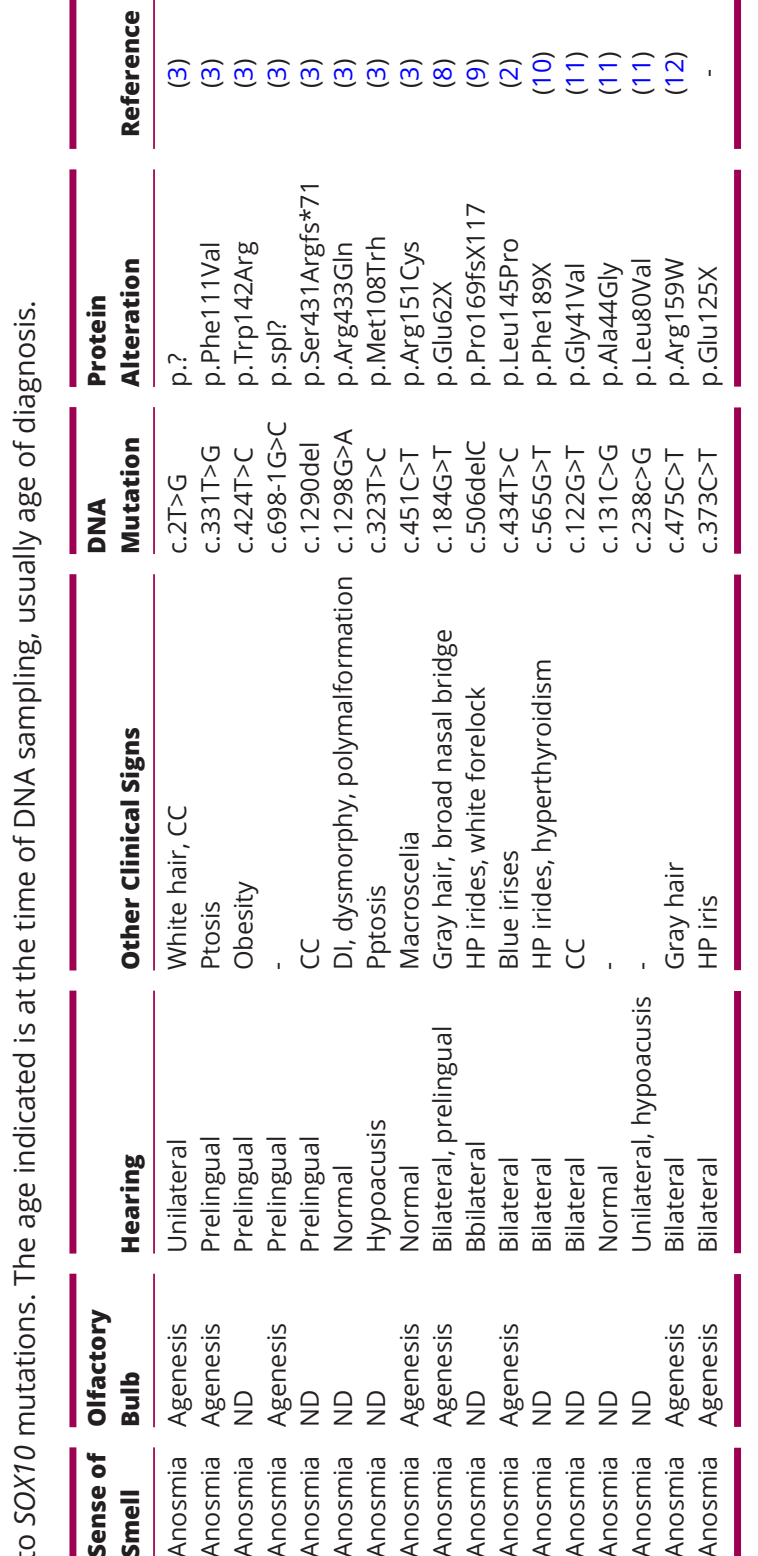

ฯ

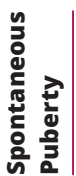

家

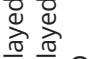

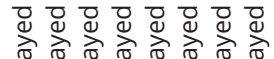

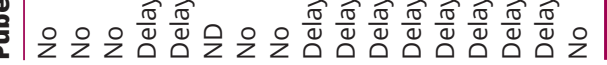

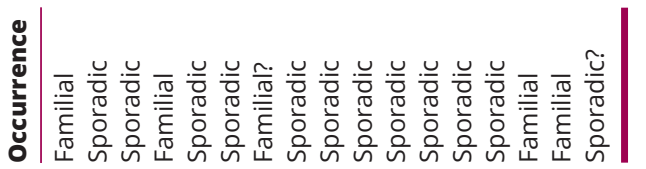

离||

:

岁|

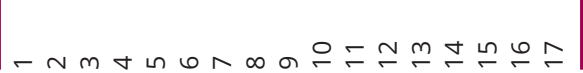

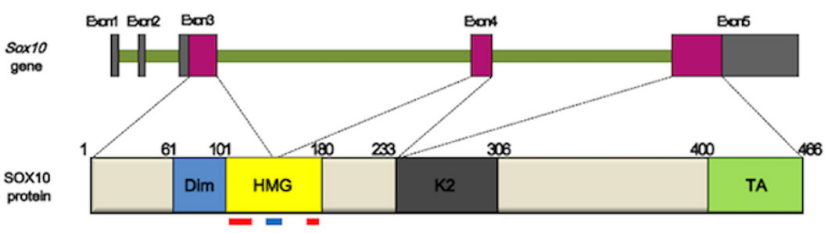

Figure 4

Schematic view of SOX10 gene and SOX10 protein. The purple areas of the soX10 gene indicate the coding region. Dim, dimerization domain; HMG, high-mobility group; K2, K2 domain; TA, transactivation domain; NLS, nuclear localization signals; NES, nuclear export sequence. Numbers refer to amino-acid residues. The red lines and the blue line under the HMG domain represent the NLS and the NES sequences, respectively.

(Applied Biosystems). Mutations were confirmed by sequencing duplicate PCR templates from separate reactions.

\section{Treatment}

He did not receive gonadotropin-replacement therapy but testosterone-replacement therapy because of the absence of desire for childbearing. Initially, he received $125 \mathrm{mg}$ testosterone enantate intramuscularly every four weeks, and the dose of testosterone has been gradually increased depending on the subjective symptoms and his blood testosterone level. After 1.5 years of dose titration, his condition became stable with the maintenance treatment: $500 \mathrm{mg}$ testosterone enantate every four to five weeks.

\section{Outcome and follow-up}

His height stopped increasing at the age of 19 years, and he has finally grown to $176 \mathrm{~cm}$. As a result of the testosterone-replacement therapy, he succeeded in avoiding being excessively tall and eunuchoid, and to show masculinity.

\section{Discussion}

Herein, we report a male KS patient with WS, who carried a novel nonsense mutation (c.373C $>\mathrm{T}$, p.Glu125X) of SOX10. The patient had deafness and a hypopigmented iris, yet no dystopia canthorum, limb anomaly, or symptoms of $\mathrm{HD}$, indicating that his manifestations match those reported for WS2. While his family members did not consent to genetic analysis, they appeared phenotypically normal, and we regarded the patient as a sporadic case.

Recently, the neural crest has been implicated in the etiology of KS, particularly in cases with hearing loss. 
Initially, mutations in $C H D 7$, which is one of the key genes in neural crest development, have been shown to account for about one-third of KS patients with deafness. Secondly, SOX10 mutations were identified in KS patients, and have been reported in 17 patients with KS in total, including the present case, to date $(2,3,8,9,10,11,12)$. As a certain degree of hearing loss was observed in 14 of the 17 patients, deafness is conceivably a consistent feature of patients with SOX10 mutations. In contrast, 8 patients showed various degrees of hypopigmentation, 3 patients showed neurological defects other than deafness, and neither limb anomaly nor HD were described (Table 2). Overall, SOX10 mutations are associated with a variety of clinical WS features.

SOX10 is a key transcription factor involved in the development and migration of neural crest cells, which differentiate into melanocytes, Schwann cells, oligodendrocytes, and peripheral glia neurons. Dysfunction of melanocytes can result in defects in melanin synthesis, leading to abnormal pigmentation of the hair, skin, and iris. In addition, it can induce congenital hearing impairment, as melanocytes are essential for inner ear development (13). Furthermore, olfactory ensheathing cells, which are required for the development of the olfactory bulb and the migration of GNRH neurons, can be deficient due to SOX10 mutations, leading to anosmia and hypogonadism (3). Therefore, SOX10 mutations can induce various developmental defects, including an overlapping phenotype of KS and WS.

SOX10 belongs to the SRY-related high-mobility group (HMG) box family of transcription factors. SOX10 consists of 466 amino acids and comprises the following domains (from N- to C-terminus): DNA-dependent dimerization domain, DNA-binding HMG domain, context-dependent transactivation domain $\mathrm{K} 2$, and main transactivation domain TA. The HMG domain possesses two distinct nuclear localization signals (NLS) and a nuclear export sequence (NES) to drive the SOX proteins into and out of the nucleus, respectively (Fig. 4) (14). Previous findings indicate that mutant SOX10 mRNA occurs in any coding exon, but the last one is recognized by the nonsensemediated RNA decay pathway and degraded; therefore, most SOX10 mutations will lead to haploinsufficiency. In contrast, if the mutation in the last coding exon occurs, a mutant protein is considered to act as a dominant negative protein impairing the function of normal SOX10, resulting in peripheral demyelinating neuropathy, central dysmyelinating leukodystrophy, WS, and HD (15). The present case carried a heterozygous nonsense substitution c.373C $>\mathrm{T}$ (p.Glu125X) in the HMG domain of SOX10, which is predicted to be pathogenic due to SOX10 haploinsufficiency. To the best of our knowledge, this nonsense mutation has never been published as a germline variant and has only been observed as a somatic variant in a melanoma sample (16). Thus, the SOX10 substitution (c.373C $>$ T, p.Glu125X) is a novel mutation that results in KS or WS.

In this case, in addition to KS and WS, the patient also presented with subclinical hypothyroidism without evidence of autoimmune thyroiditis. To date, there are no reports describing the relationship between SOX10 and hypothyroidism. As recent findings have indicated that subpopulations of neural crest cells may influence thyroid morphogenesis (17), it is possible that SOX10 is also associated with the development of the thyroid gland. Ultrasonography did not show a definite malformation of the thyroid in this case, however, disorders of neural crest development caused by SOX10 mutations might affect thyroid function. Since it is not clear whether subclinical hypothyroidism was an unrelated comorbidity, further research is required to address this issue in the future.

Patients with WS tend to be diagnosed in childhood, through recognition of congenital hearing impairment or abnormal pigmentation, whereas most KS patients are diagnosed after puberty. As KS may remain undetected in previously reported patients with WS due to SOX10 mutations, incidences of SOX10 mutations in KS patients may increase in the future.

In conclusion, we identified a novel nonsense SOX10 mutation in a patient diagnosed with KS and WS. This case substantiates the significance of SOX10 as a genetic cause of KS and WS, which possibly share a common pathway during neural crest cell development. Additional phenotypic features should be carefully evaluated in patients with KS and/or WS. Further studies are necessary to fully elucidate the clinical characteristics of SOX10 mutations.

\section{Declaration of interest}

The authors declare that there is no conflict of interest that could be perceived as prejudicing the impartiality of the research reported.

\section{Funding}

This research did not receive any specific grant from any funding agency in the public, commercial, or not-for-profit sector.

\section{Patient consent}

Written informed consent was obtained from the patient and his parents for the genetic testing and the publication of this case report and any accompanying data and images. 


\section{Author contribution statement}

T W, A T, N O, and M Y: patient management, data acquisition; T W, M T, $\mathrm{S} \mathrm{N}, \mathrm{K} \mathrm{O}, \mathrm{KE}$, and $\mathrm{S}$ I: conceptualization; $\mathrm{T} W$ : genetic testing, wrote the manuscript; $T \mathrm{~W}$ and $\mathrm{S}$ I: edited the manuscript. All authors read and approved the final manuscript.

\section{References}

1 Cangiano B, Swee DS, Quinton R \& Bonomi M. Genetics of congenital hypogonadotropic hypogonadism: peculiarities and phenotype of an oligogenic disease. Human Genetics 2021140 77-111. (https://doi.org/10.1007/s00439-020-02147-1)

2 Suzuki E, Izumi Y, Chiba Y, Horikawa R, Matsubara Y, Tanaka M, Ogata T, Fukami M \& Naiki Y. Loss-of-function SOX10 mutation in a patient with Kallmann syndrome, hearing loss, and iris hypopigmentation. Hormone Research in Paediatrics 201584 212-216. (https://doi.org/10.1159/000436965)

3 Pingault V, Bodereau V, Baral V, Marcos S, Watanabe Y, Chaoui A Fouveaut C, Leroy C, Vérier-Mine O, Francannet C, et al. Lossof-function mutations in SOX10 cause Kallmann syndrome with deafness. American Journal of Human Genetics 201392 707-724. (https://doi.org/10.1016/j.ajhg.2013.03.024)

4 Ringer J. Identification of Waardenburg syndrome and the management of hearing loss and associated sequelae: a review for the pediatric nurse practitioner. Journal of Pediatric Health Care 201933 694-701. (https://doi.org/10.1016/j.pedhc.2019.06.001)

5 Bondurand $\mathrm{N} \&$ Sham MH. The role of SOX10 during enteric nervous system development. Developmental Biology 2013382 330-343. (https://doi.org/10.1016/j.ydbio.2013.04.024)

6 Richards S, Aziz N, Bale S, Bick D, Das S, Gastier-Foster J, Grody WW, Hegde M, Lyon E, Spector E, et al. Standards and guidelines for the interpretation of sequence variants: a joint consensus recommendation of the American College of Medical Genetics and Genomics and the Association for Molecular Pathology. Genetics in Medicine 201517 405-424. (https://doi. org/10.1038/gim.2015.30)

7 Maeda K, Asai R, Maruyama K, Kurihara Y, Nakanishi T, Kurihara H \& Miyagawa-Tomita S. Postotic and preotic crania neural crest cells differently contribute to thyroid development. Developmental Biology 2016409 72-83. (https://doi.org/10.1016/j. ydbio.2015.10.026)
8 Iso M, Fukami M, Horikawa R, Azuma N, Kawashiro N \& Ogata T. SOX10 mutation in Waardenburg syndrome type II. American Journal of Medical Genetics: Part A 2008 146A 2162-2163. (https://doi. org/10.1002/ajmg.a.32403)

9 Vaaralahti K, Tommiska J, Tillmann V, Liivak N, Känsäkoski J, Laitinen EM \& Raivio T. De novo SOX10 nonsense mutation in a patient with Kallmann syndrome and hearing loss. Pediatric Research 201476 115-116. (https://doi.org/10.1038/pr.2014.60)

10 Izumi Y, Musha I, Suzuki E, Iso M, Jinno T, Horikawa R, Amemiya S, Ogata T, Fukami M \& Ohtake A. Hypogonadotropic hypogonadism in a female patient previously diagnosed as having Waardenburg syndrome due to a sox10 mutation. Endocrine 201549 553-556. (https://doi.org/10.1007/s12020-014-0434-4)

11 Wang F, Zhao S, Xie Y, Yang W \& Mo Z. De novo SOX10 nonsense mutation in a patient with Kallmann syndrome, deafness, iris hypopigmentation, and hyperthyroidism. Annals of Clinical and Laboratory Science 201848 248-252.

12 Dai W, Wu J, Zhao Y, Jiang F, Zheng R, Chen DN, Men M \& Li JD. Functional analysis of SOX10 mutations identified in Chinese patients with Kallmann syndrome. Gene 2019702 99-106. (https:// doi.org/10.1016/j.gene.2019.03.039)

13 Zhang Q, He HH, Janjua MU, Wang F, Yang YB, Mo ZH, Liu J \& Jin P. Identification of two novel mutations in three Chinese families with Kallmann syndrome using whole exome sequencing. Andrologia 2020 52 e13594. (https://doi.org/10.1111/and.13594)

14 Saleem MD. Biology of human melanocyte development, piebaldism, and Waardenburg syndrome. Pediatric Dermatology 201936 72-84. (https://doi.org/10.1111/pde.13713)

15 Chaoui A, Watanabe Y, Touraine R, Baral V, Goossens M, Pingault V $\&$ Bondurand N. Identification and functional analysis of SOX10 missense mutations in different subtypes of Waardenburg syndrome. Human Mutation 201132 1436-1449. (https://doi.org/10.1002/ humu.21583)

16 Inoue K, Khajavi M, Ohyama T, Hirabayashi S, Wilson J, Reggin JD, Mancias P, Butler IJ, Wilkinson MF, Wegner M, et al. Molecular mechanism for distinct neurological phenotypes conveyed by allelic truncating mutations. Nature Genetics 200436 361-369. (https://doi. org/10.1038/ng1322)

17 Cronin JC, Wunderlich J, Loftus SK, Prickett TD, Wei X, Ridd K, Vemula S, Burrell AS, Agrawal NS, Lin JC, et al. Frequent mutations in the MITF pathway in melanoma. Pigment Cell and Melanoma Research 200922 435-444. (https://doi.org/10.1111/j.1755148X.2009.00578.x

Received in final form 24 March 2021

Accepted 2 April 2021 